\title{
Digital PET/CT: a new intriguing chance for clinical nuclear medicine and personalized molecular imaging
}

\author{
Orazio Schillaci $^{1,2}$ (D) $\cdot$ Nicoletta Urbano ${ }^{3}$ \\ Received: 21 February 2019 / Accepted: 27 February 2019 / Published online: 11 March 2019 \\ (C) Springer-Verlag GmbH Germany, part of Springer Nature 2019
}

Clinical PET/CT is a well-known useful tool for the in vivo noninvasive quantitative imaging of physiologic and pathologic processes [1]. Actually, the key role of PET/CT in cancer staging and therapeutic responses to personalized treatments is well established; it is an essential imaging modality not only in oncology but also in neuroscience and in all the applications of molecular imaging. In recent years, there have been multiple advances in PET/CT scanners: new hardware, software, and acquisition methods to improve image quality [2]. Nevertheless, till now PET detectors have been mainly based on photomultiplier tubes (PMT), which have well-known advantages but also several limitations that affect, in particular, small lesions detection [3]. The introduction of digital detectors in PET/CT scanners may represent an important improvement in this diagnostic technology [4].

In this issue of EJNMMI, Lopez-Mora et al. [5] present a step forward in this field, comparing image quality and lesion detection capability between a digital (d) and an analog (a) $\mathrm{PET} / \mathrm{CT}$ system in 100 patients with oncologic diseases who were prospectively included in this study. The patients consecutively underwent a single day, dual imaging protocol (d and aPET/CT) after a i.v. injection of either FDG or fluorocholine. The first PET/CT was performed $60 \mathrm{~min}$ after the i.v. injection of the radiopharmaceutical, and the second imaging dataset was acquired with a mean time delay of 50 $\pm 14 \mathrm{~min}$. In the patients referred for an initial assessment $(n=$

This Editorial Commentary refers to the article https://doi.org/10.1007/ s00259-019-4260-z.

Orazio Schillaci

orazio.schillaci@uniroma2.it

1 Department of Biomedicine and Prevention, University Tor Vergata, Viale Mazzini 121, 00195 Rome, Italy

2 IRCCS Neuromed, Pozzilli, Italy

3 Nuclear Medicine, Policlinico Tor Vergata, Rome, Italy
58), the $\mathrm{dPET} / \mathrm{CT}$ was performed first, while in the patients who were evaluated for therapy monitoring, the aPET/CT was firstly acquired. Three nuclear medicine physicians evaluated image quality using a 4-point scale ( -1 , poor to 2 , excellent) and detection capability by counting the number of lesions with increased uptake of the radiopharmaceutical. In $54 \%$ of patients, dPET/CT allowed a better image quality than aPET/ $\mathrm{CT}$; in the remaining 46 patients, image quality did not significantly differ between both devices. Regarding lesion detection capability, dPET/CT was able to visualize lesions in three patients in whom the a system resulted negative; moreover, in 19 out of 80 cases which were positive at aPET/CT imaging, the $\mathrm{dPET} / \mathrm{CT}$ detected more lesions. In these 22 patients, all the lesions visualized only by means of the $\mathrm{dPET} / \mathrm{CT}$ were $<$ $1 \mathrm{~cm}$ in size: eight were in the lungs, eight in lymph-nodes, six in the liver, four in bones, and one in seminal vesicles, in the breasts and in the skin, respectively. It is worth noting that $\mathrm{dPET} / \mathrm{CT}$ changed staging in $32 \%$ of these patients ( 7 out of 22).

In the same group of 100 oncological patients, another recent study was conducted to assess whether $\mathrm{dPET} / \mathrm{CT} \mathrm{im}$ pact on the quantification of SUVmax in target lesions (the most metabolically active in each case) and in reference regions (liver and mediastinal blood pool) in comparison to aPET/CT [6]. The findings of this paper indicate that SUVmax of the target lesions and mediastinal blood pool measured by the $d$ system were significantly higher than with those obtained by the a one, whereas liver mean SUVmax did not differ between the two devices. These results were obtained in the patients in whom aPET/CT was performed first, and in those in whom dPET/CT was acquired first. This is an interesting issue, considering the impossibility of doing both studies at the same time, and so the influence of delayed increased radiopharmaceutical uptake cannot be excluded. These results suggest that the $d$ scanner has higher sensitivity than the a system, and this feature has to be taken into account in daily clinical practice before interchangeable use of either PET/CT scanner in patients' follow-up studies. 
In an initial study on dPET/CT [7], a prototype was used in 21 oncological patients and compared with an aPET system with time-of-flight (TOF). Two experienced nuclear medicine physicians firstly reviewed the two PET datasets separately for overall image quality on a 4-point scale (1, poor to 4 , excellent), and then compared the two datasets side by side for lesion conspicuity, sharpness, and diagnostic confidence using a 5 -point scale (1, much worse to 5 , much better), all aware of the scanner type. Six patients were at staging/initial diagnosis and 15 at restaging; the time delay between start of aPET and digital dPET was $22.6+12 \mathrm{~min}$. The overall image quality was scored higher with dPET (median 4) than with aPET (median 3). In a visual side-by-side comparison, lesion conspicuity, lesion sharpness and diagnostic confidence resulted better for dPET with respect to aPET. Moreover, dPET was able to identify all the lesions seen by aPET, and to detect eight additional positive sites in five out of 21 patients, leading to upstaging in two patients evaluated for initial staging. It is worth nothing that these additional lesions had a median size of $0.5 \mathrm{~cm}$ (range, $0.5-0.9 \mathrm{~cm}$ ), with a SUVmax of 1.0 (range, 0.8-2.2) for aPET and 1.5 (range, 1.2-3.4) for dPET. Based on these results, the study conclusions were that dPET in oncological patients, providing better image quality, diagnostic confidence, and accuracy than aPET, may be most beneficial in detecting and characterizing small lesions and in disease staging by contributing to upstaging.

The same authors recently reported a comparison in tumor volume delineation between dPET and aPET [8]. In the 11 patients included in the final analyses showing FDG avid lesions greater than physiologic liver uptake, the median size of the 24 measured sites was $1.6 \mathrm{~cm}$. The lesion volume at dPET was smaller at both $35 \%$ SUVmax and 50\% SUVmax thresholds compared with that of aPET, with a mean difference of $-3680.0 \mathrm{~mm}^{3}$ at $35 \%$ SUVmax and $-835.3 \mathrm{~mm}^{3}$ at $50 \%$ SUVmax. The smaller volume definition and the improved lesion conspicuity and sharpness of dPET allow this device to be more accurate in tumor rendering compared with aPET, not only for radiotherapy planning but also in prognostication and treatment monitoring. These findings open new perspectives for dPET in tumor quantification and characterization.

The physical performance of two PET systems [9], one based on new-generation detectors of digital silicon photomultipliers (SiPM) and the other one with PMT and TOF, were evaluated using NEMA NU 2 (published by the National Electrical Manufacturers Association (NEMA)), provided by the vendor for each PET/CT scanner, allowing reproducible and accepted measurement standards for evaluating the physical performance of PET systems [10]. The SiPMPET/CT demonstrated higher sensitivity, better performance under high count-rate conditions and better image quality than the conventional PMT-PET/CT system. These findings suggest that better-quality images might be achieved under routine scanning schedules with $\mathrm{dPET} / \mathrm{CT}$, thus enabling shortening acquisition time or reducing radiotracer dose. With a substantial decrease in patient exposure to radioactivity, we have an opportunity to utilize PET more broadly in non-oncologic applications, such as neuroscience, cardiovascular disease, and infection-inflammation imaging. All these benefits are very synergistic with the development of new PET radiopharmaceuticals or new applications for existing radiotracers.

A recent study has evaluated the performance of a new dPET/CT, with SiPM-based detectors with 3.2-mm lutetium oxyorthosilicate (LSO) crystals and full coverage of the scintillator area [11]. Spatial resolution, sensitivity, count-rate performance, accuracy of attenuation and scatter correction, TOF performance, and image quality have been determined. Measurements were directly compared to results from its aPET/CT predecessor, using existing literature. The dPET outperforms the aPET in every NEMA test: it shows a transverse (resp. axial) spatial resolution of $3.7 \mathrm{~mm}$ (resp. $3.8 \mathrm{~mm}$ ) at $1 \mathrm{~cm}$ offset from the center of the field of view (measured with a FDG source), compared to $4.3 \mathrm{~mm}$ (resp. $4.3 \mathrm{~mm}$ ) shown by the aPET. Furthermore, an increase in sensitivity of $70.3 \%$, a $65 \%$ higher peak noise equivalent count-rate, and a higher contrast recovery were reached with respect to the aPET; the timing resolution improved from $540 \mathrm{ps}$ on the aPET to $210 \mathrm{ps}$ on the dPET.

A pilot study [12] had compared semi-quantitative measurements between $\mathrm{d}$ and aPET/CT in 50 patients included for initial diagnosis and/or staging $(n=9)$, or subsequent treatment strategy (therapy monitoring, restaging and detection of suspected recurrence, $n=41)$. Standard aPET/ $\mathrm{CT}$ images were acquired $43.3 \pm 112.6$ min (mean $\pm \mathrm{SD}$ : $64.4 \pm 13.5$ ) after FDG injection; dPET/CT scans started at $71.2 \pm 142.2 \mathrm{~min}($ mean $\pm \mathrm{SD}: 102.1 \pm 17.4 \mathrm{~min})$ after radiopharmaceutical administration, with a delay time between beginning of aPET/CT and dPET/CT of $17.5 \pm 75.1$ (mean \pm SD: $37.7 \pm 13.9$ ). Images were reviewed and analyzed independently by two blinded nuclear medicine physicians. The dPET/CT was able to visualize all the 107 lesions seen by the standard aPET/CT, and 37 additional lesions in 14 out of 50 patients, indicating that it is more sensitive and can better detect smaller lesions. As a matter of fact, the SUVmax measurements for the 107 lesions were higher on dPET/CT compared with aPET/CT, and not correlated to the acquisition delay of dPET data.

The influence of acquisition time on image quality in a $\mathrm{dPET} / \mathrm{CT}$ scanner has been recently reported in a group of 58 subjects with cancer [13]. PET datasets were acquired in list mode, then they were reprocessed to produce these sets of sinograms: 30, 60, 90, and $120 \mathrm{~s}$ per bed. The reference standard acquisition time was 180-210 s per bed, depending on the patients' body mass index (BMI). Two experienced nuclear medicine physicians reviewed blindly all PET reconstructions for image quality, that was rated using a 5 -point scale $(1$, 
non-diagnostic to 5 , excellent) in which a score of 3 , 4 , or 5 was considered to provide diagnostic value. The average scores \pm SD of the readers were $2.61 \pm 0.83,3.70 \pm 0.92$, $4.36 \pm 0.82,4.82 \pm 0.39$, and $4.91 \pm 0.91$ for the $30,60,90$, and $120 \mathrm{~s} / \mathrm{bed}$ and at standard acquisition time, respectively. These findings suggest that, even in a patient population with average BMI $>25$, images can be acquired as fast as $90 \mathrm{~s} / \mathrm{bed}$ using the $\mathrm{dPET} / \mathrm{CT}$ and still result in very good quality (average score $>4$ ).

Because the resolution and quantitation accuracy of tracer uptake in PET are highly influenced by several factors, including the reconstruction method, Lindström et al. [14] have assessed the performance of a new penalized-likelihood reconstruction algorithm and its impact on image quality and SUV measurements on a TOF dPET/CT scanner. Data from whole-body FDG PET/CT scans of 11 patients were examined ( 8 oncologic and 3 patients studied because of suspected inflammatory disease). Two nuclear medicine physicians visually analyzed image quality, blindly rating several aspects; moreover, noise level, signal-to-noise ratio (SNR), signal-tobackground ratio (SBR), and SUV were evaluated. When compared with the standard nonregularized ordered-subsets expectation maximization (OSEM) reconstruction, penalized-likelihood reconstruction algorithm allowed an increased tumor SUVmax and an improved SNR and SBR at a matched level of noise. Furthermore, the findings of this study suggest that the acquisition time can be reduced from 3 to $2 \mathrm{~min} / \mathrm{bed}$ position when penalized-likelihood reconstruction is used instead of TOF OSEM, with a mean saving of $5 \mathrm{~min}$ for a PET scan of 5 bed positions.

The clinical implication of using the above cited reconstruction algorithm on image quality in a $\mathrm{dPET} / \mathrm{CT}$ scanner has been studied in 45 patients undergoing FDG imaging for initial lung cancer staging [15]. The quality of each reconstructed image dataset was evaluated by two readers using a 5 -point scale (1, poor to 5, excellent); thereafter, the same persons scored the images for sharpness and lesion conspicuity by means of another 5-point Likert scale. Quantitative imaging analysis was made by a third reader in a separate reading session: the SUVmax of each primary lung tumor was recorded using a standard volume of interest (VOI) tool; then, background SUVs were assessed in the right liver lobe (parenchymal organ background) and within the descending aorta (bloodpool background) at the level of the carina, with spherical VOIs. The reconstruction algorithm used allowed to obtain, when compared to OSEM, higher SUVmax of lung tumors up to $+28 \%$, and higher SBR and contrast-tobackground ratio of lung tumor, and higher SNR and contrast-to-noise ratio. This increased quantitative accuracy led to a better image quality, image sharpness, and tumor lesion conspicuity, with significant clinical implications.

The compliance of a dPET/CT system to the EANM Research Ltd. (EARL) accreditation specifications for tumour imaging with FDG has beeen reported in a phantom study using a NEMA NU2-2001 image quality phantom with six fillable spheres [16]. According to the EANM guidelines. PET using digital photon counting technology meet EARL standards when using an OSEM reconstruction with $4 \times 4 \times$ $4 \mathrm{~mm}^{3}$ voxels, no point spread function modelling and including a Gaussian post-smoothing filter of $2-4 \mathrm{~mm}$. These parameters are important for using $\mathrm{dPET} / \mathrm{CT}$ scanners in nuclear medicine research and multi-centre studies.

The reduction of FDG dose in clinical PET imaging by using SiPM detectors was assessed in 74 patients with different oncologic diseases who underwent sequential whole-body TOF-PET/CT and TOF PET/MR imaging [17]. SiPM detectors, which enable TOF in PET/MR hybrid devices, are more sensitive than the LYSO crystals of conventional TOF-PET/ $\mathrm{CT}$; therefore, this property can be used to lower the injected dose of radiopharmaceuticals. In this study, PET image quality with simulated $60 \%$ of the standard injected dose (reduction of up to $40 \%$ ) of FDG at TOF-PET/MR imaging with SiPM detectors was found to be clinically adequate and comparable to that at standard TOF-PET/CT with LYSO detectors. Thus, substantially lower radiation doses compared with those at conventional PET/CT can be achieved in PET imaging with SiPM detectors.

The above cited articles indicate that dPET/CT technology is the latest evolution of clinical PET which enables advanced molecular imaging capabilities and supports new opportunities for personalized nuclear medicine. This system demonstrates excellent performance characteristics, which lead to more precise localization of the annihilation events and contribute to reduction in partial volume effect, thus making high definition PET imaging feasible. This improved image quality can be used to optimize administered radioactivity and/or scan duration. In clinical PET/CT imaging, these factors should be considered and harmonized to generate the best possible image quality and a low radiation dose to the patient as well as reasonable acquisition times to get a fast workflow. Rapid PET/CT imaging acquisition might be particularly important in pediatric patients (reducing anaesthesia or sedation time) or for sick patients who cannot remain still for long periods.

In the near future, in addition to $\mathrm{dPET} / \mathrm{CT}$, the effective sensitivity of PET could be enhanced by increasing the geometric coverage to encompass the whole body. As a matter of fact, although the radiopharmaceutical distribution is systemic, current PET systems contain only a small portion of the body within the field of view. For studies in which the entire body or multiple organs are of interest, the world's first totalbody PET/CT scanner is currently being constructed to achieve a sensitivity increase of a factor of about 40 for imaging the whole body [18].

In conclusion, PET image quality has not fundamentally changed over the last two decades, but now dPET/CT seems to be a new relevant opportunity not only for this issue, but 
also for improving lesion detectability and diagnostic confidence. How these encouraging initial findings could be translated rapidly into the patient care paradigm, and improve it, is a matter for further studies in larger populations. We really hope that $\mathrm{dPET} / \mathrm{CT}$ can expand current, and more importantly open new, translational health-care applications for nuclear medicine molecular imaging in the study of human disease as part of the developing vision of precision, personalized, systems-based medicine [19].

\section{Compliance with ethical standards}

This article does not contain any studies with human participants or animals performed by any of the authors.

Conflict of interest Both authors declare that they have no conflicts of interest.

Publisher's note Springer Nature remains neutral with regard to jurisdictional claims in published maps and institutional affiliations.

\section{References}

1. Mittra E, Quon A. Positron emission tomography/computed tomography: the current technology and applications. Radiol Clin N Am. 2009;47:147-60.

2. Slomka PJ, Pan T, Germano G. Recent advances and future progress in PET instrumentation. Semin Nucl Med. 2016;46:5-19.

3. van der Vos CS, Koopman D, Rijnsdorp S, Arends AJ, Boellaard R, van Dalen JA, et al. Quantification, improvement, and harmonization of small lesion detection with state-of-the-art PET. Eur J Nucl Med Mol Imaging. 2017;44(Suppl 1):4-16.

4. Zhang J, Maniawski P, Knopp MV. Performance evaluation of the next generation solid-state digital photon counting PET/CT system. EJNMMI Res. 2018;8:97. https://doi.org/10.1186/s13550-0180448-7.

5. López-Mora DA, Flotats A, Fuentes-Ocampo F, Camacho V, Fernández A, Ruiz A, et al. Comparison of image quality and lesion detection between digital and analog PET/CT. Eur J Nucl Med Mol Imaging. 2019. https://doi.org/10.1007/s00259-019-4260-z.

6. Fuentes-Ocampo F, López-Mora DA, Flotats A, Paillahueque G, Camacho V, Duch J, et al. Digital vs. analog PET/CT: intra-subject comparison of the SUVmax in target lesions and reference regions. Eur J Nucl Med Mol Imaging. 2019. https://doi.org/10.1007/ s00259-018-4256-0.
7. Nguyen NC, Vercher-Conejero JL, Sattar A, Miller MA, Maniawski PJ, Jordan DW, et al. Image quality and diagnostic performance of a digital PET prototype in patients with oncologic diseases: initial experience and comparison with analog PET. J Nucl Med. 2015;56:1378-85.

8. Nguyen NC, Vercher-Conejero J, Faulhaber P. Tumor volume delineation: a pilot study comparing a digital positron-emission tomography prototype with an analog positron-emission tomography system. World J Nucl Med. 2019;18:45-51.

9. Wagatsuma K, Miwa K, Sakata M, Oda K, Ono H, Kameyama M, et al. Comparison between new-generation SiPM-based and conventional PMT-based TOF-PET/CT. Phys Med. 2017;42:203-10.

10. National Electrical Manufacturers Assoc. Performance measurements of positron emission tomographs. NEMA standards publication NU 2-2012. Rosslyn: National Electrical Manufacturers Association; 2012.

11. van Sluis JJ, de Jong J, Schaar J, Noordzij W, van Snick P, Dierckx $\mathrm{R}$, et al. Performance characteristics of the digital biograph vision PET/CT system. J Nucl Med. 2019. https://doi.org/10.2967/ jnumed.118.215418.

12. Baratto L, Park SY, Hatami N, Davidzon G, Srinivas S, Gambhir SS, et al. 18F-FDG silicon photomultiplier PET/CT: a pilot study comparing semi-quantitative measurements with standard PET/CT. PLoS One. 2017;12(6):e0178936.

13. Sonni I, Baratto L, Park S, Hatami N, Srinivas S, Davidzon G, et al. Initial experience with a SiPM-based PET/CT scanner: influence of acquisition time on image quality. EJNMMI Phys. 2018;5:9. https:// doi.org/10.1186/s40658-018-0207-x.

14. Lindström E, Sundin A, Trampal C, Lindsjö L, Ilan E, Danfors T, et al. Evaluation of penalized-likelihood estimation reconstruction on a digital time-of-flight PET/CT scanner for (18)F-FDG wholebody examinations. J Nucl Med. 2018;59:1152-8.

15. Messerli M, Stolzmann P, Egger-Sigg M, Trinckauf J, D’Aguanno $\mathrm{S}$, Burger IA, et al. Impact of a Bayesian penalized likelihood reconstruction algorithm on image quality in novel digital PET/CT: clinical implications for the assessment of lung tumors. EJNMMI Phys. 2018;5:27. https://doi.org/10.1186/s40658-018-0223-x.

16. Koopman D, Groot Koerkamp M, Jager PL, Arkies H, Knollema S, Slump CH, et al. Digital PET compliance to EARL accreditation specifications. EJNMMI Phys. 2017;4:9. https://doi.org/10.1186/ s40658-017-0176-5.

17. Sekine T, Delso G, Zeimpekis KG, de Galiza Barbosa F, Ter Voert EEGW, Huellner M, et al. Reduction of (18)F-FDG dose in clinical PET/MR imaging by using silicon photomultiplier detectors. Radiology. 2018;286:249-59.

18. Cherry SR, Jones T, Karp JS, Qi J, Moses WW, Badawi RD. Totalbody PET: maximizing sensitivity to create new opportunities for clinical research and patient care. J Nucl Med. 2018;59:3-12.

19. Schillaci O, Urbano N. Personalized medicine: a new option for nuclear medicine and molecular imaging in the third millennium. Eur J Nucl Med Mol Imaging. 2017;44:563-6. 